\title{
Reflex control of arterial pressure and heart rate in short-term streptozotocin diabetic rats
}

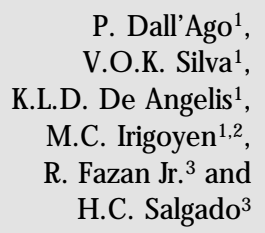

P. Dall'Ago ${ }^{1}$,

V.O.K. Silva ${ }^{1}$,

K.L.D. De Angelis ${ }^{1}$,

M.C. Irigoyen ${ }^{1,2}$,

R. Fazan Jr. ${ }^{3}$ and

H.C. Salgado ${ }^{3}$

\author{
1Departamento de Fisiologia, Instituto de Ciências Básicas da Saúde, \\ Universidade Federal do Rio Grande do Sul, Porto Alegre, RS, Brasil \\ 2Unidade de Hipertensão e Divisão de Experimentação, Instituto do Coração, \\ Faculdade de Medicina, Universidade de São Paulo, São Paulo, SP, Brasil \\ ${ }^{3}$ Departamento de Fisiologia, Faculdade de Medicina de Ribeirão Preto, \\ Universidade de São Paulo, Ribeirão Preto, SP, Brasil
}

\section{Correspondence \\ H.C. Salgado \\ Departamento de Fisiologia \\ FMRP, USP \\ Av. Bandeirantes, 3900 \\ 14049-900 Ribeirão Preto, SP \\ Brasil \\ Fax: + 55-16-633-0017 \\ E-mail: hcsalgad@ fmrp.usp.br}

Research supported by CNPq, CAPES, FAPERGS, FAPESP and PRONEX.

Received March 5, 2001

Accepted March 18, 2002

\section{Abstract}

Impaired baroreflex sensitivity in diabetes is well described and has been attributed to autonomic diabetic neuropathy. In the present study conducted on acute (10-20 days) streptozotocin (STZ)-induced diabetic rats we examined: 1) cardiac baroreflex sensitivity, assessed by the slope of the linear regression between phenylephrine- or sodium nitroprusside-induced changes in arterial pressure and reflex changes in heart rate (HR) in conscious rats; 2) aortic baroreceptor function by means of the relationship between systolic arterial pressure and aortic depressor nerve (ADN) activity, in anesthetized rats, and 3) bradycardia produced by electrical stimulation of the vagus nerve or by the $i v$ injection of methacholine in anesthetized animals. Reflex bradycardia $(-1.4 \pm 0.1 \mathrm{vs}-1.7 \pm 0.1 \mathrm{bpm} / \mathrm{mmHg})$ and tachycardia $(-2.1 \pm 0.3 v \mathrm{~s}$ $-3.0 \pm 0.2 \mathrm{bpm} / \mathrm{mmHg}$ ) were reduced in the diabetic group. The gain of the ADN activity relationship was similar in control $(1.7 \pm 0.1 \% \mathrm{max} /$ $\mathrm{mmHg})$ and diabetic $(1.5 \pm 0.1 \% \mathrm{max} / \mathrm{mmHg})$ animals. The HR response to vagal nerve stimulation with 16,32 and $64 \mathrm{~Hz}$ was 13,16 and $14 \%$ higher, respectively, than the response of STZ-treated rats. The HR response to increasing doses of methacholine was also higher in the diabetic group compared to control animals. Our results confirm the baroreflex dysfunction detected in previous studies on short-term diabetic rats. Moreover, the normal baroreceptor function and the altered HR responses to vagal stimulation or methacholine injection suggest that the efferent limb of the baroreflex is mainly responsible for baroreflex dysfunction in this model of diabetes.

\section{Introduction}

Autonomic neuropathy is the most serious complication of diabetes in terms of morbidity and mortality (1). It has been frequently reported that diabetes can affect both somatic (mainly sensitive) and autonomic nerves. The arterial baroreceptors are an im-
Key words

- Baroreflex

- Baroreceptors

- Streptozotocin

- Vagus nerve portant system that acts against wide oscillations in arterial pressure (AP), acting on both the sympathetic and parasympathetic limbs of the autonomic nervous system $(2,3)$. A number of studies have dealt with cardiac baroreflex function in humans (4), as well as in experimental diabetes $(5,6)$. Although most studies point to baroreflex dysfunction, it is 
still controversial whether both components (tachycardia and bradycardia) of the baroreflex are affected and whether or not the time course of diabetes affects the degree of baroreflex dysfunction. Reflex tachycardia due to a decrease in AP was found to be attenuated as early as five days after the onset of experimental diabetes induced by streptozotocin (STZ) in rats (6). However, the reflex bradycardia due to an increase in AP was found to be preserved in this early stage of experimental diabetes. Conversely, reflex bradycardia is impaired in chronic alloxan diabetes in rabbits while reflex tachycardia is preserved in this model of diabetes (5).

Most of the reports showing an impairment of baroreflex sensitivity have attributed it to autonomic neuropathy, while the afferent arm of the baroreflex, i.e., the arterial (carotid and aortic) baroreceptors, has received much less attention. McDowell et al. (7) demonstrated that the slope of the pressure-aortic nerve activity curve and the slope of the pressure-renal nerve activity curve were not affected by chronic alloxan diabetes in rabbits. These findings indicate that the baroreceptor function as well as the reflex control of renal sympathetic activity are preserved in this model of experimental diabetes. Fazan Jr. et al. (8) also showed normal aortic baroreceptor function in rats with chronic STZ-induced diabetes.

In the present investigation we examined the baroreflex control of heart rate (HR) in rats with short-term diabetes induced by STZ, as well as the aortic baroreceptor function and the HR response to vagal stimulation or muscarinic agonist injections.

\section{Material and Methods}

\section{Animal preparation}

The experiments were performed on male Wistar rats weighing 200-250 g, housed in individual cages with free access to water and food. Diabetes was induced by a single intravenous injection of STZ $(50 \mathrm{mg} / \mathrm{kg}$; Sigma, St. Louis, MO, USA) after an overnight fast (8-10 h). Control rats received only vehicle (10 mM citrate buffer, $\mathrm{pH} 4.5)$ after a similar fasting period. Data were obtained 10 to 20 days after STZ or citrate buffer administration.

\section{Basal hemodynamics and baroreflex}

Twenty-four hours before the experiments, control $(\mathrm{N}=11)$ and diabetic $(\mathrm{N}=11)$ rats, lightly anesthetized with ether, were implanted with catheters into the femoral artery and vein for AP measurement and drug administration, respectively. On the day of the experiment, the arterial catheter of the conscious freely moving rat was connected to a pressure transducer (P23Gb, Gould-Statham, Oxnard, CA, USA) and pulsatile AP was sampled $(1 \mathrm{kHz})$ for approximately $30 \mathrm{~min}$ with a personal computer equipped with an analog-to-digital interface (Dataq Instruments, Akron, OH, USA). After the basal recording of AP, the animals received increasing doses of phenylephrine $(0.25$ to $32 \mu \mathrm{g} / \mathrm{kg})$ or sodium nitroprusside ( 0.05 to $1.6 \mu \mathrm{g} / \mathrm{kg}$ ) given in intravenous bolus injections $(0.1 \mathrm{ml})$. An appropriate interval between doses was allowed for AP and HR to return to basal levels. The values of diastolic and systolic arterial pressure (SAP) were detected with Advanced CODAS, Dataq software and beat by beat values of mean arterial pressure (MAP) and HR were calculated. The maximal response of MAP to phenylephrine or sodium nitroprusside, and the corresponding maximal reflex change in HR were collected for each dose of the drug. The slope of the linear regression calculated on the basis of reflex changes in HR related to the induced changes in MAP was used as the index of baroreflex sensitivity.

\section{Vagal nerve stimulation and bradycardic responses to methacholine}

The bradycardia produced by electrical 
stimulation $(5 \mathrm{~V}, 2 \mathrm{~ms}, 2$ to $64 \mathrm{~Hz}$, for $10 \mathrm{~s})$ of the right vagus nerve was studied in rats anesthetized with thiopental $(35 \mathrm{mg} / \mathrm{kg}, i v)$. The interval between stimulations was determined on the basis of the time required for HR to return to basal levels. Body temperature was maintained at $37^{\circ} \mathrm{C}$ by external heating.

After vagal stimulation, the sensitivity of the muscarinic receptors of the heart was tested by evaluating HR responses to intravenous injections of increasing doses $(5,7.5$ and $10 \mu \mathrm{g} / \mathrm{kg}$ ) of methacholine.

\section{Baroreceptor recording}

In additional control $(\mathrm{N}=14)$ and diabetic ( $\mathrm{N}=17)$ groups of rats, whole aortic nerve activity was recorded as described elsewhere $(8,9)$. Briefly, under sodium pentobarbital (40 $\mathrm{mg} / \mathrm{kg}$, ip) anesthesia the aortic nerve was isolated and placed on a bipolar stainless steel pair of electrodes. Nerve activity was amplified (Princeton Applied Research Amplifier, model 113, Princeton, NJ, USA) and monitored on an oscilloscope (Tektronix, model 5113, Beaverton, OR, USA) associated with a loudspeaker. Action potentials that exceeded a threshold level set right above the noise level of each nerve activity were counted with a nerve traffic analyzer (University of Iowa Bioengineering, Iowa City, IA, USA) every 200 ms. Integrated nerve activity was sampled (100 $\mathrm{Hz}$ ) with a personal computer simultaneously with pulsatile carotid pressure. The baroreceptor firing range was assessed by rapid changes (20 to $30 \mathrm{~s}$ ) in AP due to withdrawal and reinfusion of blood into the femoral artery of the rats. To avoid the influence of hysteresis, only the values obtained during the increase in pressure (rate of $6-7 \mathrm{mmHg} / \mathrm{s}$ ) were used. Aortic nerve activity (spikes/s) was normalized (\% of maximal activity) and plotted against SAP to obtain pressure-nerve activity curves. Using a nonlinear regression based on the Levemberg-Marquadt algorithm (Marquadt, 1964) we calculated the maximum gain of the pressure-nerve activity curve and the SAP at $50 \%$ of maximal nerve activity $\left(\mathrm{SAP}_{50}\right)$ as the parameters of baroreceptor function.

\section{Blood analysis}

Immediately after the experiments, blood was collected from the arterial catheter to measure blood glucose and insulin by a colorimetric enzymatic test and by radioimmunoassay, respectively.

\section{Statistical analysis}

Data from pressure-nerve activity curves, vagal stimulation and methacholine injections were processed by multivariate analysis of variance (MANOVA) for repeated measures. If differences were observed a post hoc test (Newman-Keuls or Tukey) was employed. The unpaired Student $t$-test was used to compare the other parameters between control and diabetic rats. Changes were considered significant at $\mathrm{P}<0.05$.

\section{Results}

All rats given STZ exhibited severe hyperglycemia $(447 \pm 49$ vs $126 \pm 3 \mathrm{mg} / \mathrm{dl}, \mathrm{P}=$ $0.001)$ and insulinopenia (16 \pm 1 vs $57 \pm 11$ $\mu \mathrm{U} / \mathrm{ml}, \mathrm{P}=0.002$ ) associated with a decrease in body weight $(200 \pm 8 v s 268 \pm 5 \mathrm{~g}$, $\mathrm{P}=0.001)$ as compared to control rats.

Resting HR (322 \pm 5 vs $341 \pm 6$ bpm; $\mathrm{P}=$ $0.03)$ and resting MAP $(93 \pm 2$ vs $104 \pm 3$ $\mathrm{mmHg} ; \mathrm{P}<0.01$ ) were significantly reduced in diabetic rats.

The reflex bradycardia elicited by phenylephrine $(-1.4 \pm 0.1$ vs $-1.7 \pm 0.1 \mathrm{bpm} /$ $\mathrm{mmHg} ; \mathrm{P}=0.03$ ) as well as the reflex tachycardia elicited by sodium nitroprusside $(-2.1$ \pm 0.3 vs $-3.0 \pm 0.2 \mathrm{bpm} / \mathrm{mmHg} ; \mathrm{P}=0.02$ ) were significantly reduced in the diabetic group as indicated by the slope of the linear regression relating changes in HR to changes in MAP (Figure 1).

The HR response to vagal nerve stimula- 
Figure 1. Average linear regression calculated between phenylephrine or sodium nitroprusside changes in arterial pressure and reflex heart rate in control $(\mathrm{N}=$ $11)$ and diabetic $(\mathrm{N}=11)$ rats. $* \mathrm{P}<0.05$ compared to control (Student t-test). tion with 16,32 and $64 \mathrm{~Hz}$ was 13,16 and $14 \%$ higher than in STZ-treated rats when compared to control. The HR response to 2, 4 and $8 \mathrm{~Hz}$ was similar in normal and diabetic rats (Figure 2A).

The HR response to increasing doses of methacholine $(5,7.5$ and $10 \mu \mathrm{g} / \mathrm{kg})$ was significantly higher in the diabetic group (-152 $\pm 29,-192 \pm 13,-213 \pm 11$ vs $-73 \pm 20,-124$ $\pm 26,-157 \pm 18 \mathrm{bpm}$ in controls, respectively; $\mathrm{P}<0.05$ ), see Figure 2B.

$\mathrm{SAP}_{50}$ did not differ between diabetic $(123 \pm 3 \mathrm{mmHg}$ ) and control $(128 \pm 2 \mathrm{mmHg})$ rats. The gain of the pressure-nerve activity curve was also similar in control $(1.7 \pm 0.1 \%$
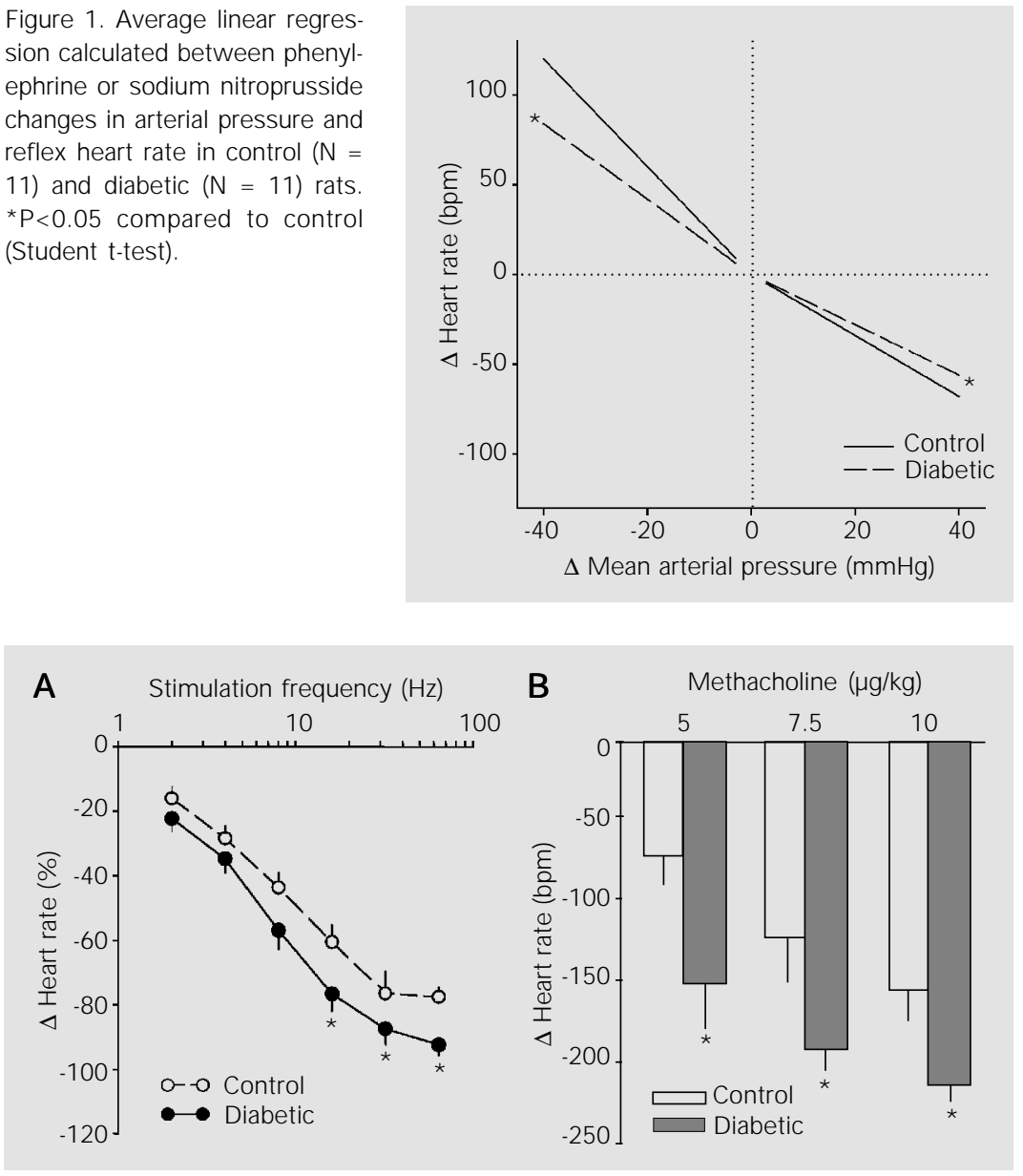

Figure 2. Effect of electrical stimulation of the right vagal nerve $(A)$ on heart rate in control $(N$ $=11$ ) and diabetic rats $(\mathrm{N}=11)$. $\mathrm{B}$, Heart rate responses produced by increasing doses of methacholine $(5,7.5$ and $10 \mu \mathrm{g} / \mathrm{kg})$ in control and diabetic rats. Data are reported as means \pm SEM. $* P<0.05$ compared to control (multivariate analysis of variance followed by Tukey post-test). $\max / \mathrm{mmHg})$ and diabetic $(1.5 \pm 0.1 \% \mathrm{max} /$ $\mathrm{mmHg}$ ) animals. The pressure (aortic depressor nerve) activity curve for each group is shown in Figure 3. As indicated by the $\mathrm{SAP}_{50}$ and the gain, the curve for STZ-treated animals was similar to that for control rats.

\section{Discussion}

The high blood glucose and low insulin levels presented by STZ-treated rats confirm the efficacy of STZ in producing diabetes in rats.

Basal levels of AP and HR were reduced in STZ-treated rats. The literature has reported the occurrence of hypertension (10), normotension (11) or hypotension $(12,13)$ in experimental diabetes. A reduction in resting HR is usually accompanied by a reduction in AP in STZ-induced diabetes (13). The mechanisms underlying the association of diabetes and bradycardia are not known, but may reflect changes in the electrophysiological properties of the sino-atrial node as indicated by a depressed spontaneous activity of the pacemaker observed in isolated hearts (14). A possible mechanism to explain the hemodynamic changes observed in diabetic rats may be similarly related to alterations in autonomic control. Indeed, we have previously demonstrated that sympathetic and parasympathetic tone to the heart was reduced in short-term experimental diabetes (12). These changes were accompanied by changes in AP and HR variability and were prevented by treatment with insulin (15).

Impairment of cardiac baroreflex in diabetic patients (4) and experimental models of diabetes has been demonstrated in a number of studies $(5,7)$.

We may hypothesize that this impairment is a consequence of the reduced sensitivity of sensory baroreceptor endings to AP changes or a consequence of a dysfunction in central baroreflex integration. In addition, an alteration of efferent pathways, including 
vagal efferent neurons, or changes in the responsiveness of muscarinic receptors to acetylcholine may also be taken into consideration.

An interesting finding of the present study was that short-term (10 to 20 days) STZinduced diabetes showed an impairment of the reflex control of HR associated with normal baroreceptor function. In addition, the bradycardia induced by electrical vagal stimulation or methacholine administration was enhanced in diabetic rats. Moreover, the resting bradycardia and hypotension observed in the present study were similar to those obtained in previous ones.

The preserved baroreceptor function during short-term (10 to 20 days) diabetes observed in the present study agrees with previous observations of normal baroreceptor function in rats with chronic STZ-induced diabetes (8) or in rabbits with alloxan-induced diabetes (7). Therefore, the attenuated HR response due to baroreceptor activation (increase in AP due to phenylephrine) or deactivation (decrease in AP due to sodium nitroprusside) in short-term diabetes cannot be related to changes in the afference of the reflex.

Studies in humans have clearly demonstrated that the baroreflex-mediated bradycardia in response to an increase in AP is impaired in diabetic subjects (16-18). In contrast, the reflex tachycardia induced by a decrease in AP is preserved (18) or impaired (13). The relative role played by the sympathetic and parasympathetic nervous system in these changes is not completely understood.

Animal models used to investigate the mechanism(s) of baroreflex dysfunction in diabetes have provided different results. Reflex bradycardia due to the increase in AP has been found to be normal (6), reduced (19) or enhanced (20). The differences in baroreflex responses reported by different authors may be due to different animal models, drugs used and time course of develop- ment of diabetes. In fact, we observed that 5 days after STZ administration the reflex tachycardia was reduced while the reflex bradycardia was not (6). However, 15 days after STZ administration we observed an attenuation of both responses (13). The present study confirms these previous findings, suggesting that in conscious rats the time course of diabetes may play a role in baroreflex dysfunction. In addition, Fazan Jr. et al. (21) showed a progressive reduction in AP and HR variability with the progress of diabetes in rats.

Although the parasympathetic tone evaluated by pharmacological blockade was depressed as early as at 5 days of STZ-induced diabetes (12), only the sympathetic control of baroreflex-mediated changes in HR (tachycardia) was reduced at that time. However, the impairment in baroreflex-mediated bradycardia in response to an increase in AP observed in the present study may indicate that a parasympathetic derangement was attenuating the reflex control of HR in diabetes.

The bradycardia elicited by electrical stimulation of vagal efferents in the range of 16 to $64 \mathrm{~Hz}$ induced a greater response in

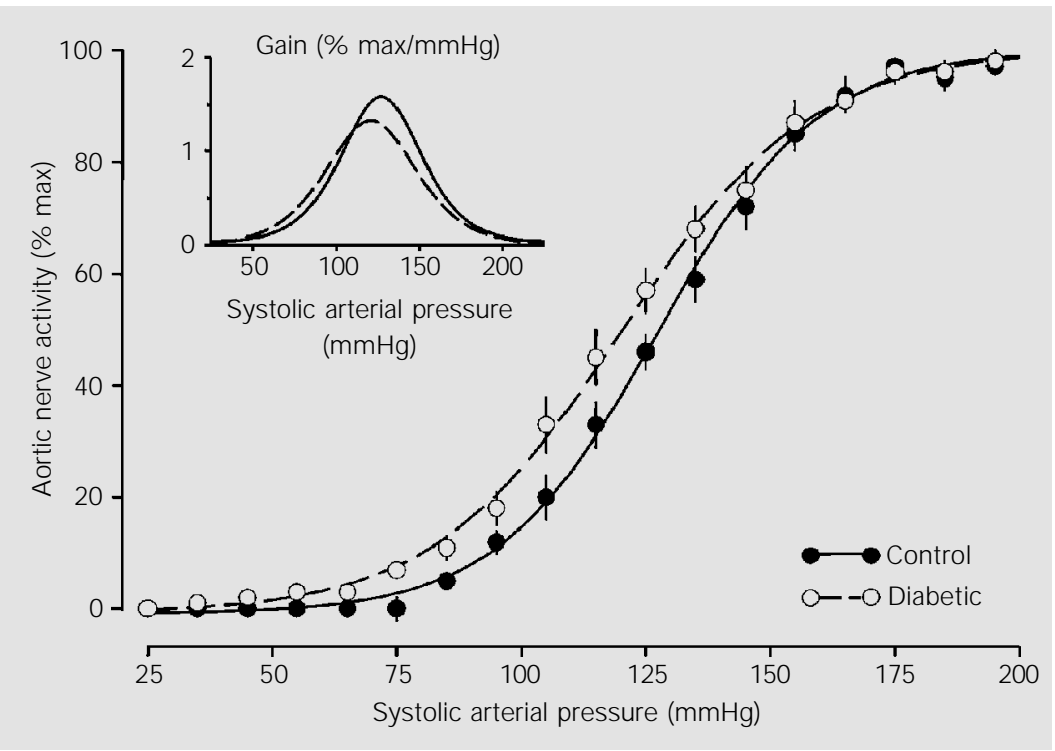

Figure 3. Pressure nerve activity curve expressed as percentage of maximal activity versus mean arterial pressure using logistic sigmoidal regression in control $(\mathrm{N}=14)$ and diabetic rats $(\mathrm{N}=17)$. Inset shows the gain of the relationship in both groups. 
diabetic rats compared to control. However, at lower frequencies of stimulation (i.e., 2 to $8 \mathrm{~Hz}$ ) we did not find any difference. These results suggest that the vagal fibers heading to the heart are not responsible for the reduced reflex bradycardia in diabetic rats. An efficient vagal discharge may induce a greater bradycardia in STZ-induced diabetic rats. Moreover, the greater bradycardia observed in diabetic rats in response to methacholine could be explained by several possible derangements such as a possible change in the number and/or affinity of the muscarinic receptors in the heart of the diabetic rats.

The study of McDowell et al. (7) showed no differences in the response to vagal electrical stimulation at frequencies up to $16 \mathrm{~Hz}$. In contrast, we did not observe differences in the lower frequency range. It is possible that these differences may be attributed to the experimental models used, i.e., rats treated with STZ versus rabbits treated with alloxan. Moreover, the bradycardia obtained in the present study was greater in response not only to the higher frequency range of stimulation but also to the agonist of the muscarinic receptors.

The reduced baroreflex tachycardia observed in diabetic rats may be related to a decreased sympathetic tone to the heart. Moreover, the changes observed in vagal tone may be contributing to the sympatheticmediated reflex tachycardia since changes in sympathetic function at different levels of parasympathetic tone have been demonstrated (12).

The altered HR responses to vagal stimulation or methacholine injection with normal baroreceptor function suggest that the efferent limb of the baroreflex is the main factor responsible for baroreflex dysfunction in this model of diabetes.

\section{Acknowledgments}

The authors acknowledge the technical assistance of Jaci A. Castania.

\section{References}

1. Ewing DJ, Campbell IW \& Clarke BF (1980). The natural history of diabetic autonomic neuropathy. Quarterly J ournal of Medicine, 49: 95-108.

2. Ganguly PK, Beamish RE, Dhalla KS, Innes IR \& Dhalla NS (1987). Norepinephrine storage, distribution, release in diabetic cardiomyopathy. American J ournal of Physiology, 252: E734-E739.

3. Lund DD, Subieta AR, Pardini BJ \& Chang KSK (1992). Alterations in cardiac parasympathetic indices in STZ-induced diabetic rats. Diabetes, 41: 160-166.

4. Eckeberg DL, Harkins SW, Fritsch JM, Musgrave GE \& Gardner DF (1986). Baroreflex control of plasma norepinephrine and heart period in healthy subjects and diabetic patients. J oumal of Clinical Investigation, 78: 366-374.

5. McDowell TS, Chapleau MW, Hajduczok $G \&$ Abboud FM (1994). Baroreflex dysfunction in diabetes mellitus, I: selective impaiment of parasympathetic control of heart rate. American J ournal of Physiology, 266: H235-H243.
6. Maeda $\mathrm{CY}$, Fernandes TG, Lulhier $\mathrm{F}$ \& Irigoyen MC (1995). Streptozotocin diabetes modifies arterial pressure and baroreflex sensitivity in rats. Brazilian J ournal of Medical and Biological Research, 28: 497501.

7. McDowell TS, Hajduczok G, Abboud FM \& Chapleau MW (1994). Baroreflex dysfunction in diabetes mellitus. II: Site of baroreflex impaiment in diabetic rabbits. American J ournal of Physiology, 266: $\mathrm{H} 244-\mathrm{H} 249$.

8. Fazan J r R, Ballejo G, Salgado MCO, Moraes MFD \& Salgado HC (1997). Heart rate variability and baroreceptor function in chronic diabetic rats. Hypertension, 30 : 632-635.

9. Dias da Silva VJ , Vargas da Silva S, Salgado MCO \& Salgado HC (1994). Chronic converting enzyme inhibition facilitates baroreceptor ressetting to hypertensive levels. Hypertension, 23: I-68-I-72.

10. Katayama S \& Lee J B (1985). Hypertension in experimental diabetes mellitus, renin-prostaglandin interaction. Hyperten- sion, 7: 554-561.

11. Hicks KK, Seifen E, Stimers J R \& Kennedy RH (1997). Diabetes with and without ketoacidosis on right atrial pacemaker rate and autonomic responsiveness. American J oumal of Physiology, 273: H1888-H1893.

12. Maeda CY, Fernandes TG, Timm H \& Irigoyen MC (1995). Autonomic dysfunction in short-term experimental diabetes. Hypertension, 26: 1000-1004.

13. Dall'Ago P, Femandes TG, Machado UF, Bello AA \& Irigoyen MC (1997). Baroreflex and chemoreflex dysfunction in streptozotocin-diabetic rats. Brazilian J ournal of Medical and Biological Research, 30: 119-124.

14. Akiyama N, Okomura $\mathrm{K}$, Watanabe $\mathrm{Y}$, Hashimoto $\mathrm{H}$, Ito $\mathrm{T}$, Ogawa $\mathrm{K} \&$ Satake $\mathrm{T}$ (1989). Altered acetylcholine and norepinephrine concentrations in diabetic rat heart. Diabetes, 38: 231-236.

15. Schaan BD, Maeda CY, Timm HB, Medeiros S, Moraes RS, Ferlin E, Femandes TG, Ribeiro J P, Schmid $\mathrm{H}$ \& Irigoyen MC (1997). Time course of changes in heart 
rate and blood pressure variability in streptozotocin-induced diabetic rats treated with insulin. Brazilian J ournal of Medical and Biological Research, 30: 1081-1086.

16. Hilsted J (1982). Pathophysiology in diabetic autonomic neurophathy: cardiovascular, hormonal, and metabolic studies. Diabetes, 31: 730-737.

17. Page MM \& Watkins PJ (1978). Cardiorespiratory arrest and diabetic autonomic neuropathy. Lancet, 1: 15-16.

18. Homma S, Yamazaki $Y \&$ Karakida $T$
(1993). Blood pressure and heart rate relationships during cervical sympathetic and vagus nerve stimulation in streptozotocin diabetic rats. Brain Research, 629: 342-344.

19. Van Buren $T$, Vleeming $W$, Krutzen $M M$, Van de Kuil T, Gipsen WH \& De Wildt DJ (1998). Vascular responses of isolated mesenteric resistance and basilar arteries from short- and long-term diabetic rats. Naunyn-Schmiedebergs Archives of Pharmacology, 358: 663-670.
20. Chang KSK \& Lund DD (1986). Alterations in the baroreceptor reflex control of heart rate in streptozotocin diabetic rats. J ournal of Molecular and Cellular Cardiology, 18: 617-624.

21. Fazan J r R, Dias da Silva VJ, Ballejo G \& Salgado HC (1999). Power spectra of arterial pressure and heart rate in streptozotocin-induced diabetes in rats. J oumal of Hypertension, 17: 489-495. 